Supporting Information for:

\title{
Evaluating UiO-66 Metal-Organic Framework (MOF) Nanoparticles as Acid-Sensitive Carriers for Pulmonary Drug Delivery Applications
}

Bader M. Jarai, ${ }^{1,}{ }^{\dagger}$ Zachary Stillman, ${ }^{1}{ }^{\dagger}$ Lucas Attia, ${ }^{1}$ Gerald E. Decker, ${ }^{2}$ Eric D. Bloch ${ }^{2}$ Catherine A. Fromen ${ }^{1, *}$

1) Department of Chemical and Biomolecular Engineering, University of Delaware, Newark, DE 19716

2) Department of Chemistry and Biochemistry, University of Delaware, Newark, DE 19716

\author{
$\dagger$ Equal contributions \\ *corresponding author. \\ cfromen@udel.edu
}

150 Academy St.

Newark, DE 19716

(302) 831-3649 


\section{Table of Contents}

Artificial Lysosomal Fluid (ALF) Composition $\quad$ S3

Powder X-Ray Diffraction (PXRD) Data $\quad$ S4

TGA Combustion Analysis Plots $\quad$ S5

$\begin{array}{ll}\text { BET Isotherm Data } & \text { S7 }\end{array}$

RhB Loading (0.5:1 to 4:1 Incubation Ratios) $\quad$ S8

$\begin{array}{ll}\text { Aerosol Characterization Table } & \text { S9 }\end{array}$

NGI Deposition Profiles $\quad$ S10

4-hour Cell Viability $\quad$ S12

Trypan Blue Quenching $\quad$ S13

4-hour Inflammatory Cytokines $\quad$ S14

Flow Cytometry Gating $\quad$ S16

$\begin{array}{ll}\text { References } & \text { S16 }\end{array}$ 
Artificial Lysosomal Fluid (ALF) Composition

Table S1: Composition of artificial lysosomal fluid. ${ }^{1}$

\begin{tabular}{|c|c|}
\hline Component & Concentration $\mathbf{( g / L )}$ \\
\hline $\mathrm{NaCl}_{2}$ & 3.21 \\
\hline $\mathrm{Na}_{2} \mathrm{HPO}_{4}$ & 0.071 \\
\hline Trisodium citrate dihydrate & 0.077 \\
\hline $\mathrm{Glycine}$ & 0.059 \\
\hline $\mathrm{NaOH}$ & 6.0 \\
\hline Citric Acid & 20.8 \\
\hline Calcium chloride dihydrate & 0.128 \\
\hline $\mathrm{Na}_{2} \mathrm{SO}_{4}$ & 0.039 \\
\hline Magnesium chloride hexahydrate & 0.05 \\
\hline Disodium tartrate & 0.09 \\
\hline Sodium lactate & 0.085 \\
\hline Sodium pyruvate & 0.172 \\
\hline
\end{tabular}




\section{Powder X-Ray Diffraction (PXRD) Data}

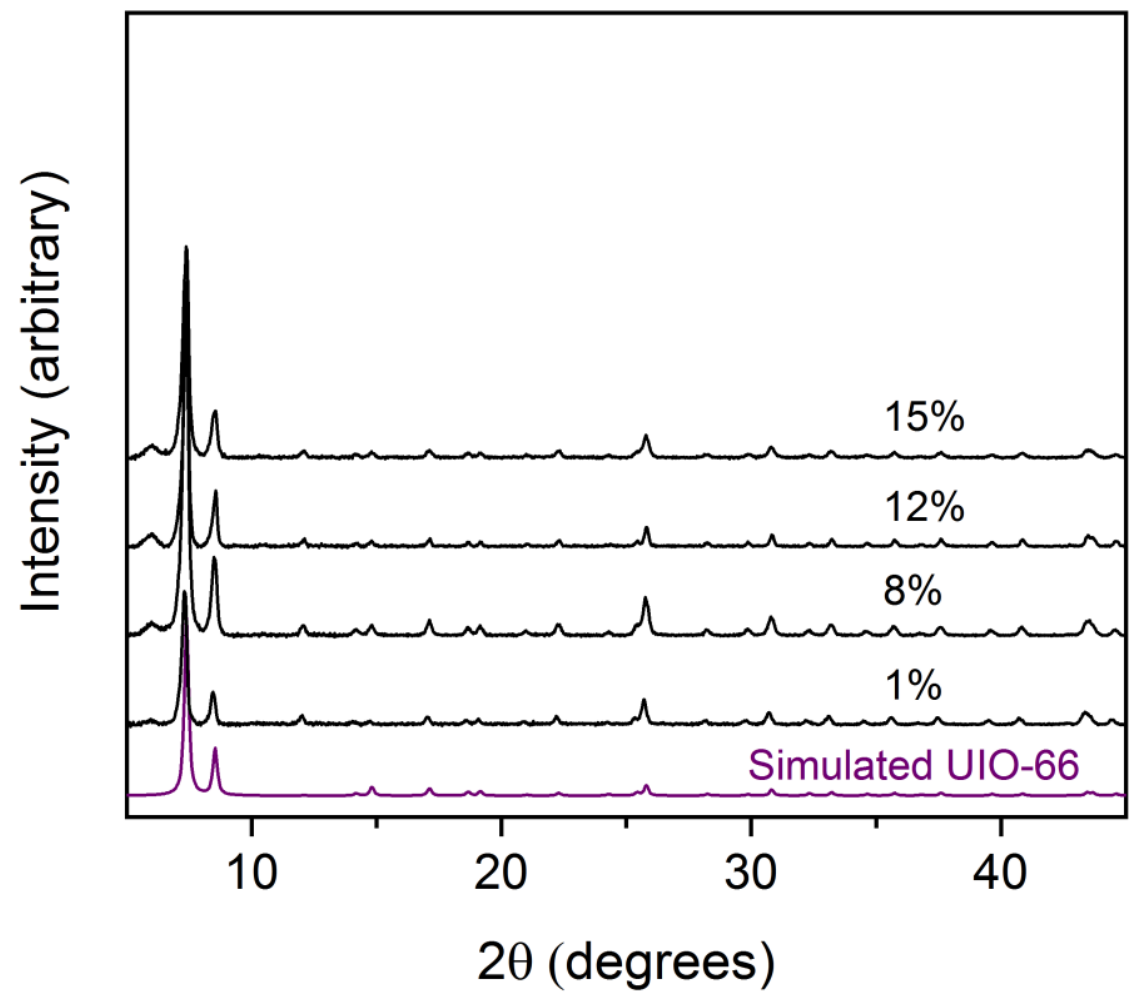

Figure S1: Powder x-ray diffraction patterns for $1 \%, 8 \%, 12 \%$, and $15 \%$ defective UiO-66 NPs. 


\section{TGA Combustion Analysis Plots}

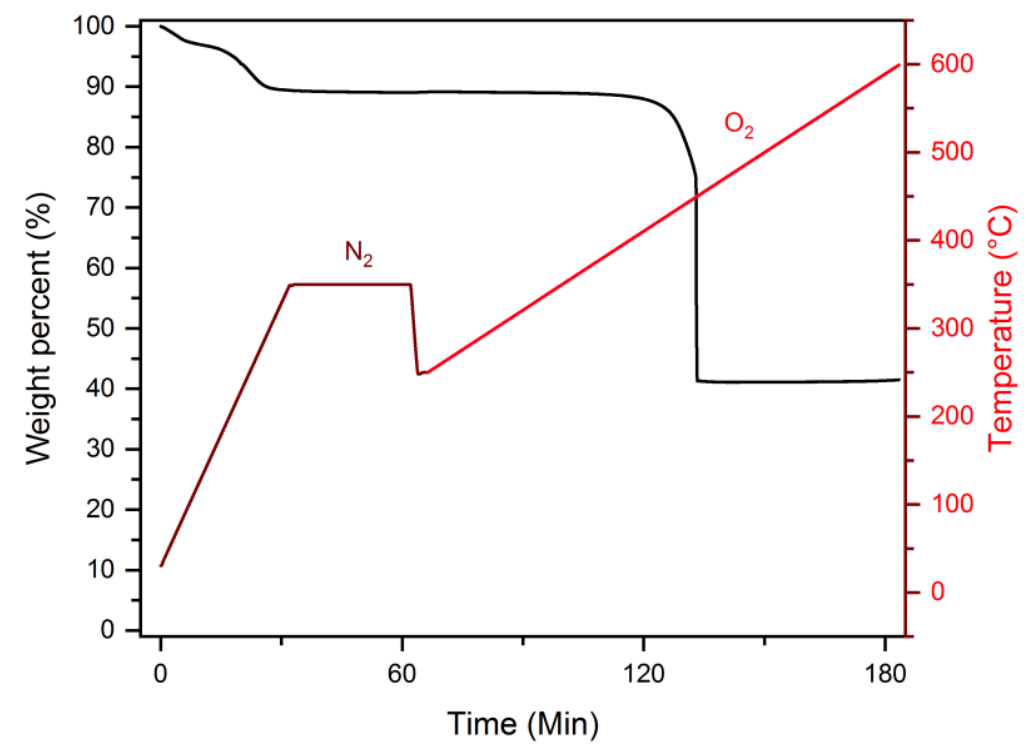

Figure S2: Thermogravimetric analysis testing for defectiveness of $1 \%$ defectiveness UiO-66.

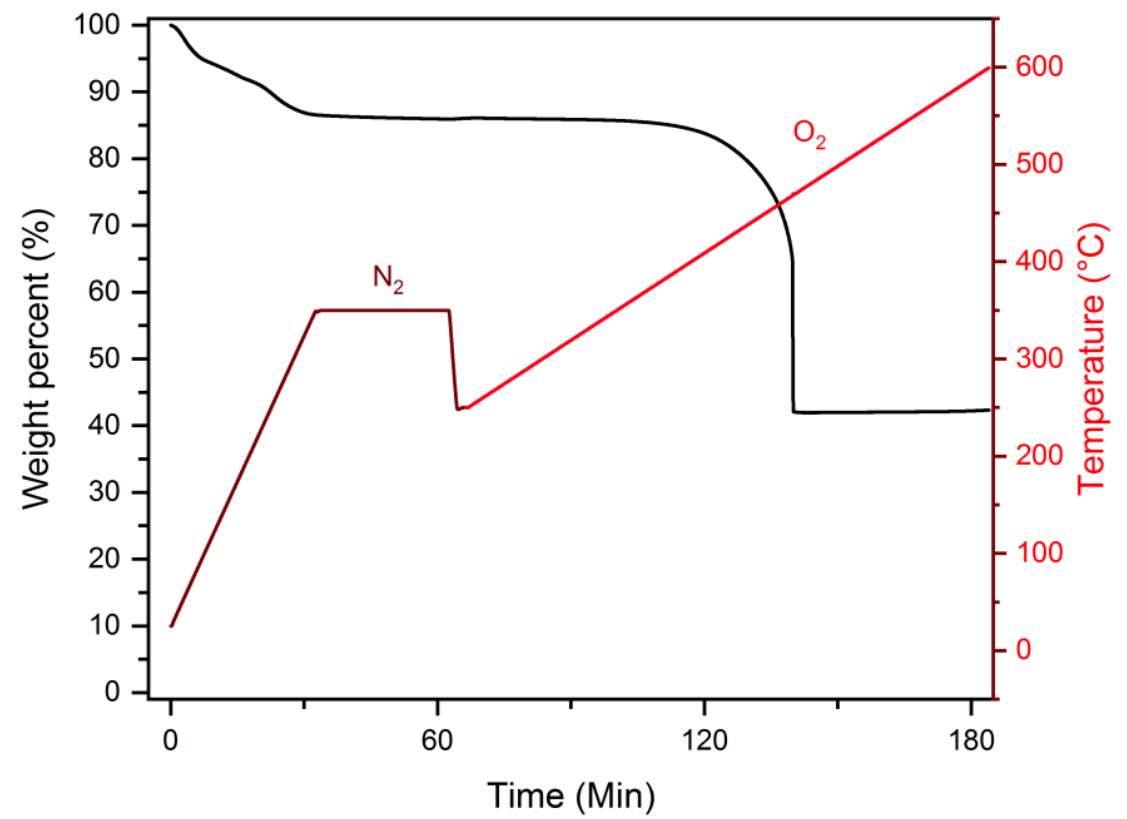

Figure S3: Thermogravimetric analysis testing for defectiveness of $8 \%$ defectiveness UiO-66. 


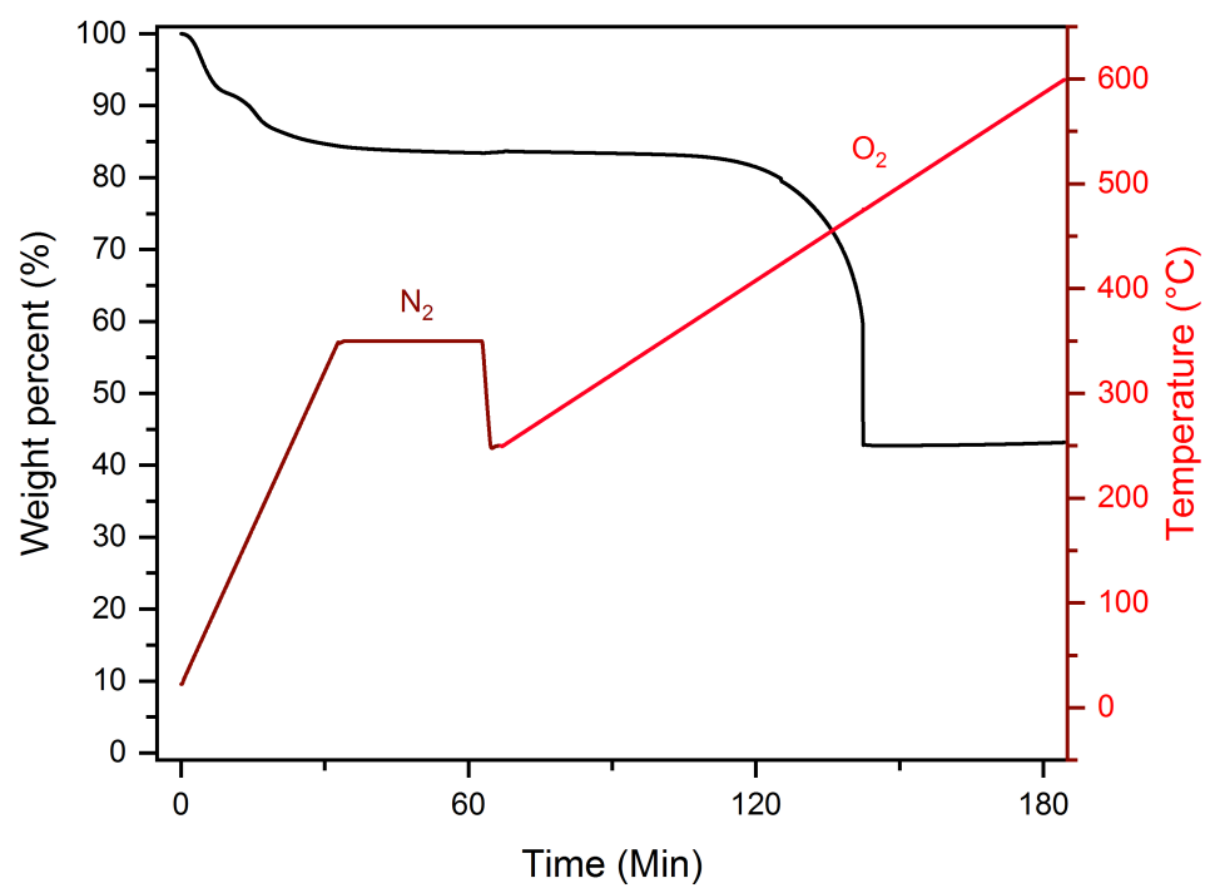

Figure S4: Thermogravimetric analysis testing for defectiveness of $12 \%$ defectiveness UiO-66.

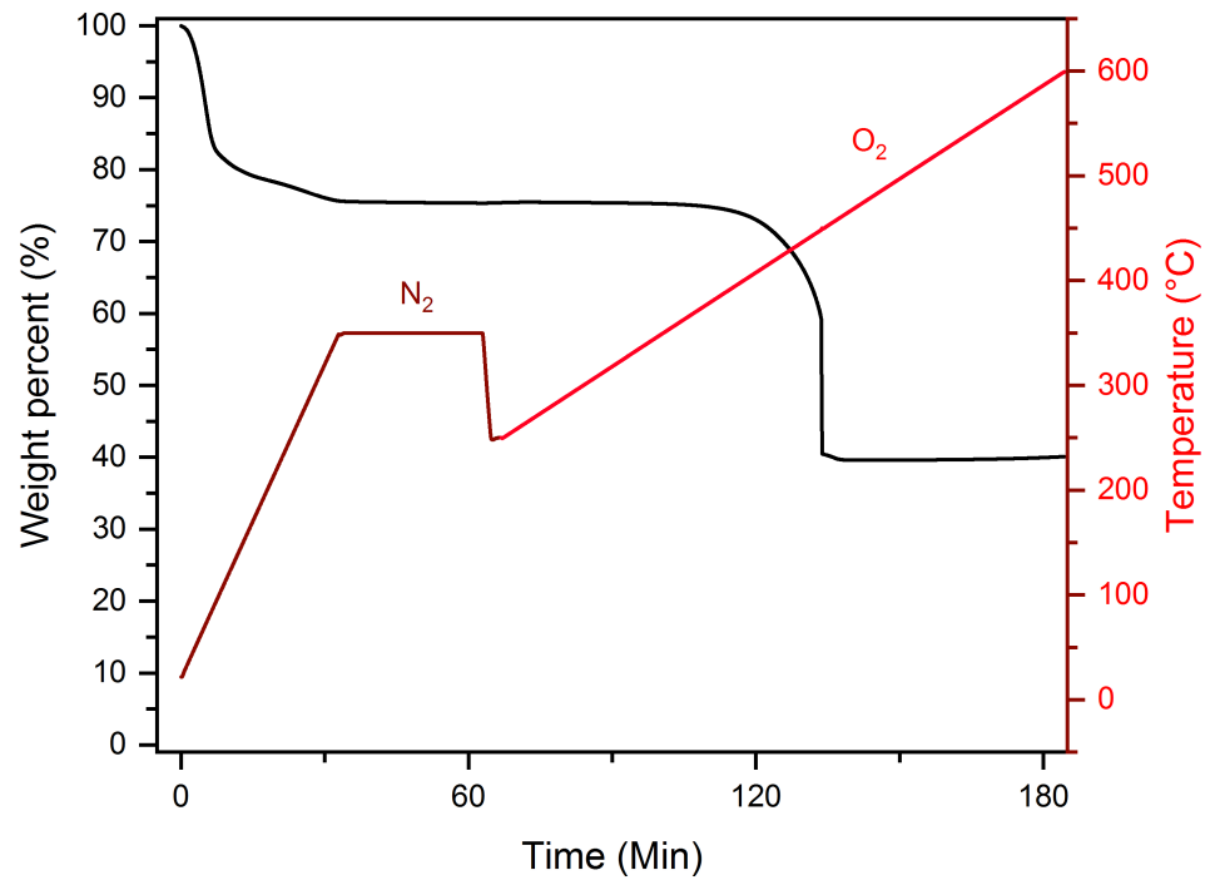

Figure S5: Thermogravimetric analysis testing for defectiveness of 15\% defectiveness UiO-66. 
BET Isotherm Data

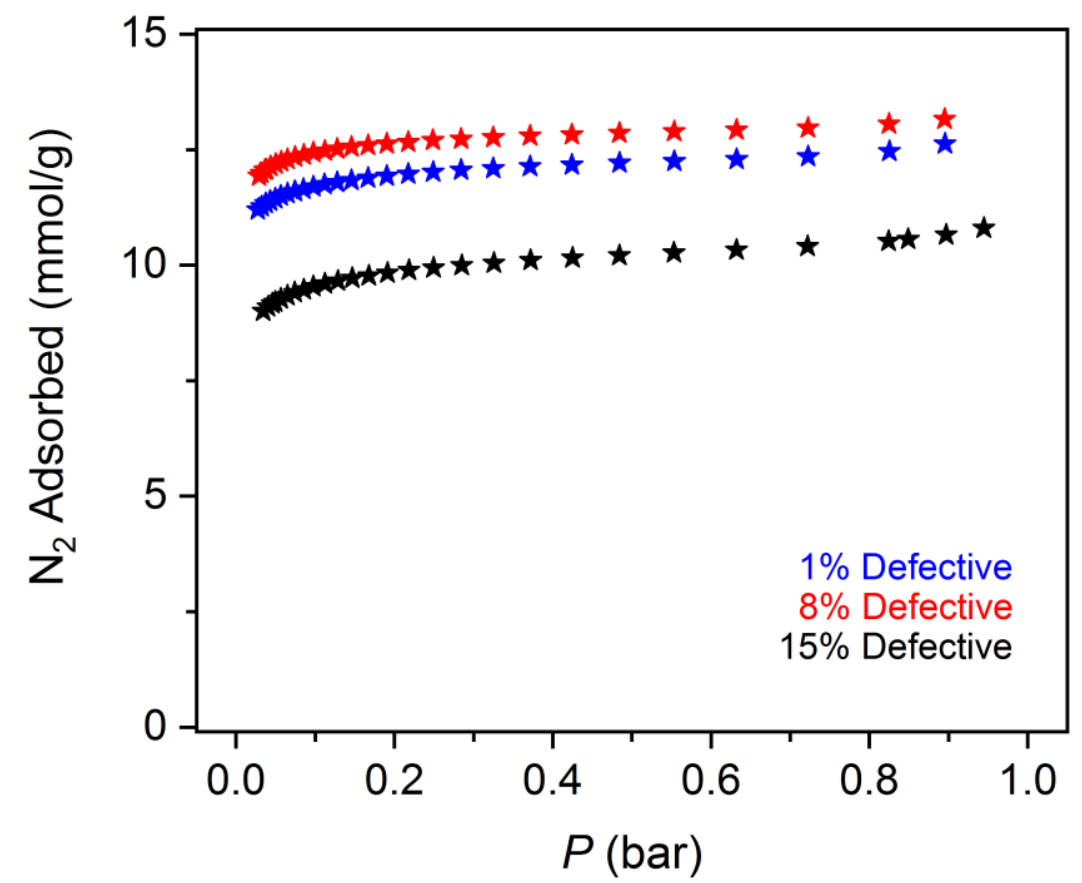

Figure S6: $\mathrm{N}_{2}$ adsorption at $77 \mathrm{~K}$ for samples at different defectiveness levels.

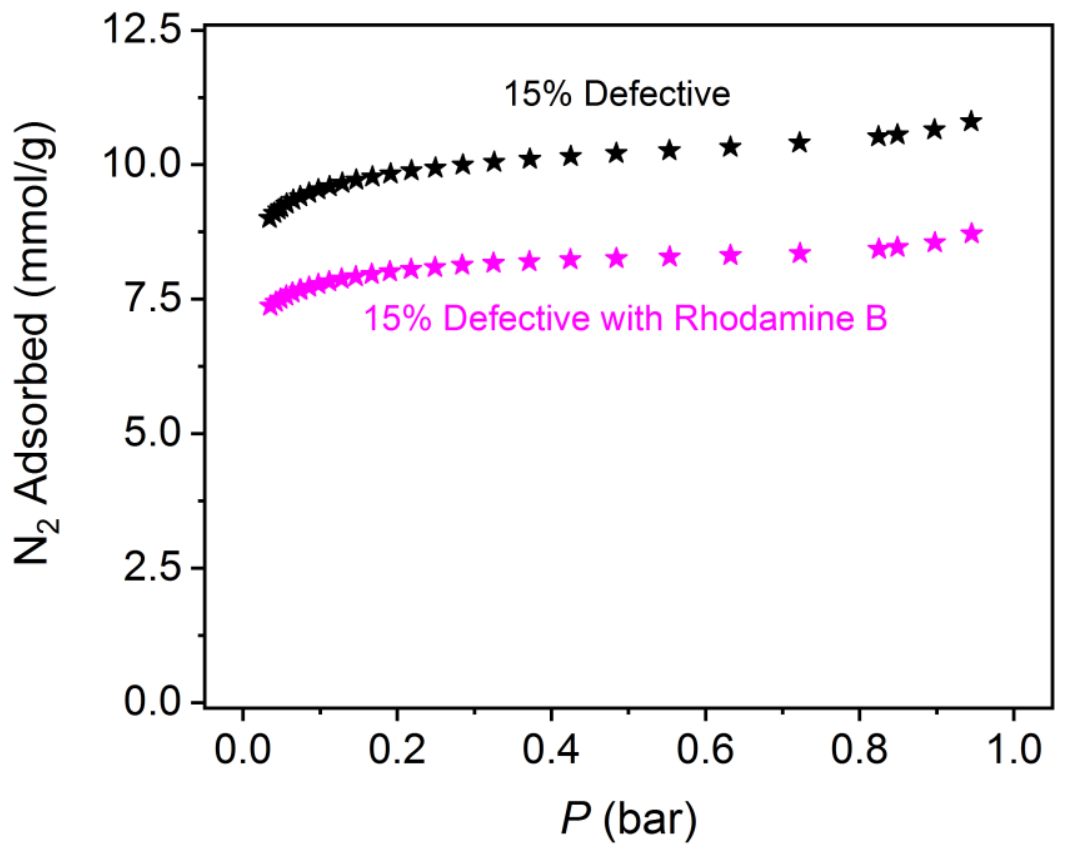

Figure S7: $\mathrm{N}_{2}$ adsorption at $77 \mathrm{~K}$ for $15 \%$ defective UiO-66 with and without Rhodamine $\mathrm{B}$ (RhB). 
RhB Loading (0.5:1 to 4:1 Incubation Ratios)

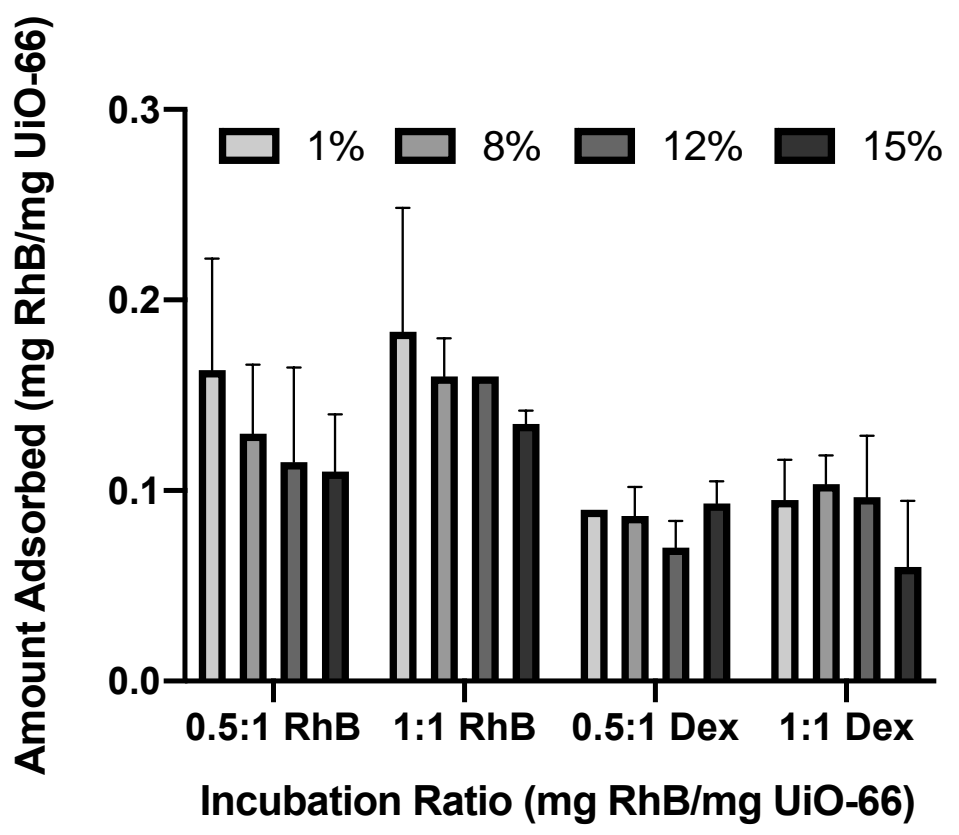

Figure S8: Loading of RhB into UiO-66 NPs. Loading amounts of RhB cargo for 1\%, 8\%, 12\%, and $15 \%$ defective UiO-66 NP samples at varied incubation ratios. Bars represent the mean, and error bars represent standard deviation $(n=3)$. 


\section{Aerosol Characterizations Table}

Table S2: Table of aerosol characterizations of UiO-66 nanoparticles of varying defectiveness (1\% to $15 \%$ ). The table lists the determined values of the mean mass aerodynamic diameter (MMAD) and geometric size distribution (GSD) for aerosolization experiments of unloaded and cargo-loaded UiO-66 NPs in a collision jet nebulizer (CJN) and the properties for aerosolization using a dry powder inhaler (DPI). The table also lists the calculated emitted dose (ED) and fine particle fraction (FPF).

\begin{tabular}{|c|c|c|c|c|c|c|c|c|}
\hline $\begin{array}{c}\text { Defectiveness } \\
(\boldsymbol{\%})\end{array}$ & $\begin{array}{c}\text { CJN } \\
\text { Unloaded } \\
\text { MMAD } \\
(\boldsymbol{\mu m})\end{array}$ & $\begin{array}{c}\text { CJN } \\
\text { Unloaded } \\
\text { GSD }\end{array}$ & $\begin{array}{c}\text { CJN } \\
\text { Loaded } \\
\text { MMAN } \\
(\boldsymbol{\mu m})\end{array}$ & $\begin{array}{c}\text { CJN } \\
\text { Loaded } \\
\text { GSD }\end{array}$ & $\begin{array}{c}\text { DPI } \\
\text { MMAD } \\
(\boldsymbol{\mu m})\end{array}$ & $\begin{array}{c}\text { DPI } \\
\text { GSD }\end{array}$ & ED & FPF \\
\hline $\mathbf{1}$ & $0.40 \pm 0.03$ & $1.32 \pm$ & $0.26 \pm$ & $2.16 \pm$ & $1.40 \pm$ & $5.04 \pm$ & $0.95 \pm$ & $0.75 \pm$ \\
& & 0.06 & 0.02 & 0.13 & 0.13 & 0.50 & 0.02 & 0.04 \\
\hline $\mathbf{8}$ & $0.36 \pm 0.03$ & $1.81 \pm$ & $0.24 \pm$ & $2.015 \pm$ & $1.16 \pm$ & $2.57 \pm$ & $0.94 \pm$ & $0.82 \pm$ \\
& & 0.54 & 0.05 & 0.13 & 0.27 & 0.09 & 0.01 & 0.03 \\
\hline $\mathbf{1 2}$ & $0.38 \pm 0.04$ & $1.51 \pm$ & $0.32 \pm$ & $1.62 \pm$ & $1.05 \pm$ & $6.15 \pm$ & $0.94 \pm$ & $0.81 \pm$ \\
& & 0.14 & 0.03 & 0.21 & 0.03 & 1.14 & 0.04 & 0.06 \\
\hline $\mathbf{1 5}$ & $0.61 \pm 0.09$ & $2.37 \pm$ & $0.32 \pm$ & $1.75 \pm$ & $1.33 \pm$ & $0.96 \pm$ & $0.96 \pm$ & $0.81 \pm$ \\
& & 0.21 & 0.05 & 0.06 & 0.13 & 0.02 & 0.02 & 0.01 \\
\hline
\end{tabular}


NGI Deposition Profiles

\section{1\% Defective Deposition Profile}

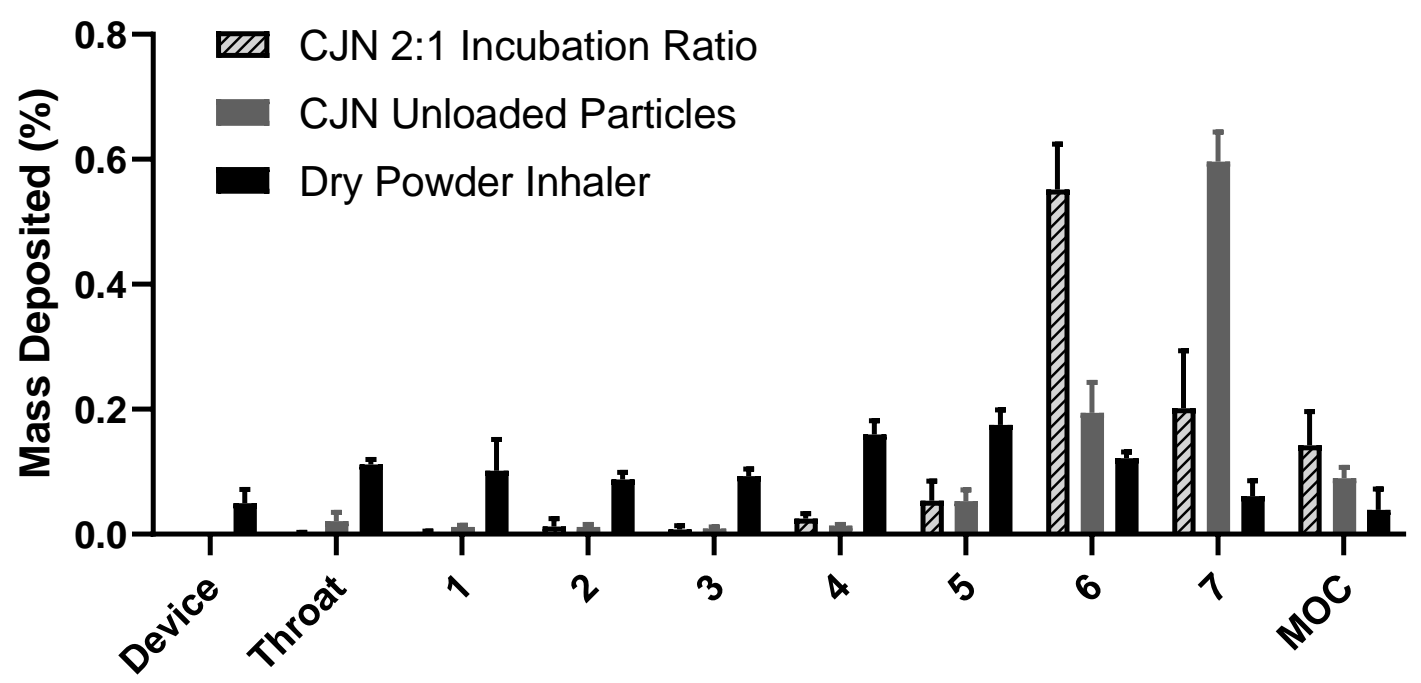

Cascade Impactor Plate

Figure S9: Mass deposition profiles for 1\% defective UiO-66 using a Next Generation Impactor.

The profiles are shown above for aerosolization with the CJN for loaded and unloaded NPs as well as for aerosolization using a DPI.

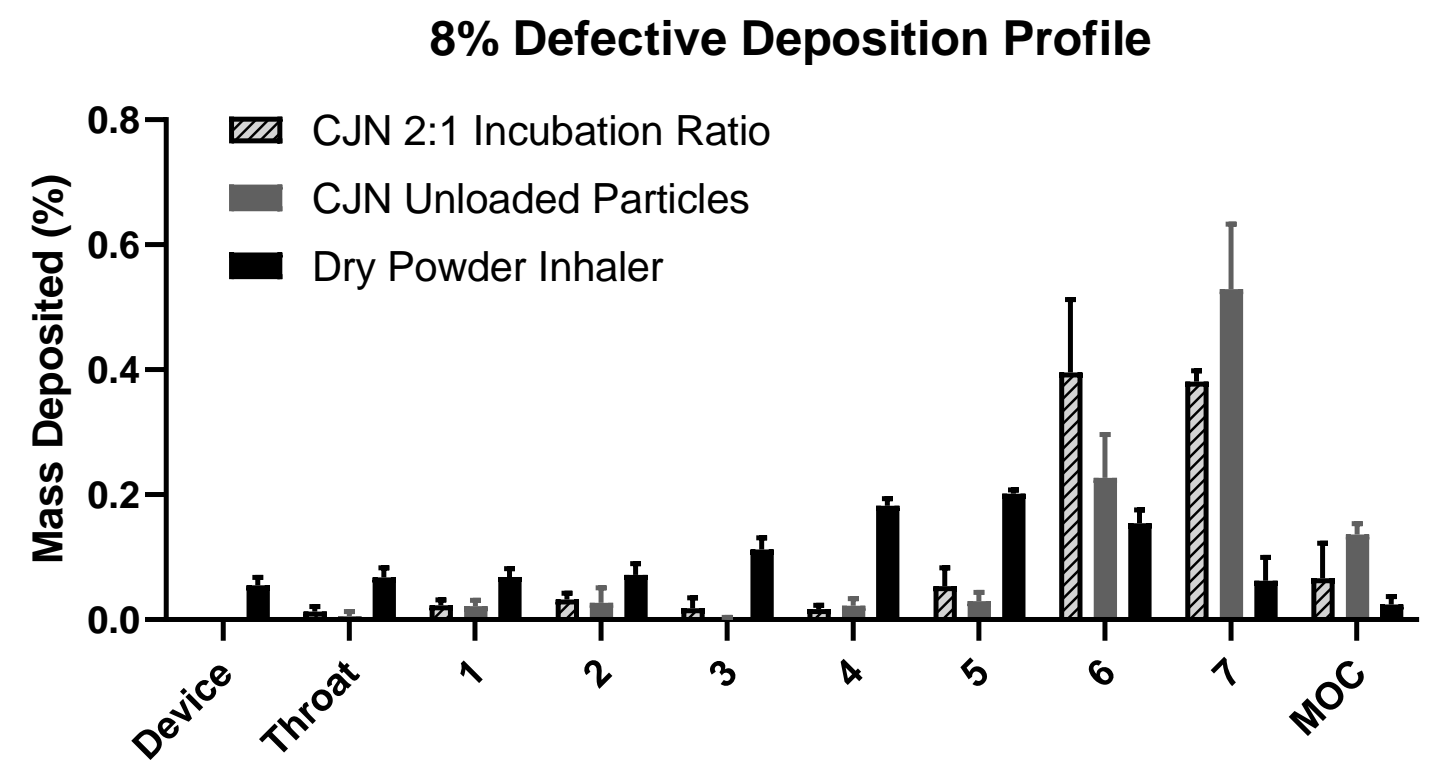

Cascade Impactor Plate

Figure S10: Mass deposition profiles for 8\% defective UiO-66 using a Next Generation Impactor. The profiles are shown above for aerosolization with the CJN for loaded and unloaded NPs as well as for aerosolization using a DPI. 


\section{2\% Defective Deposition Profile}

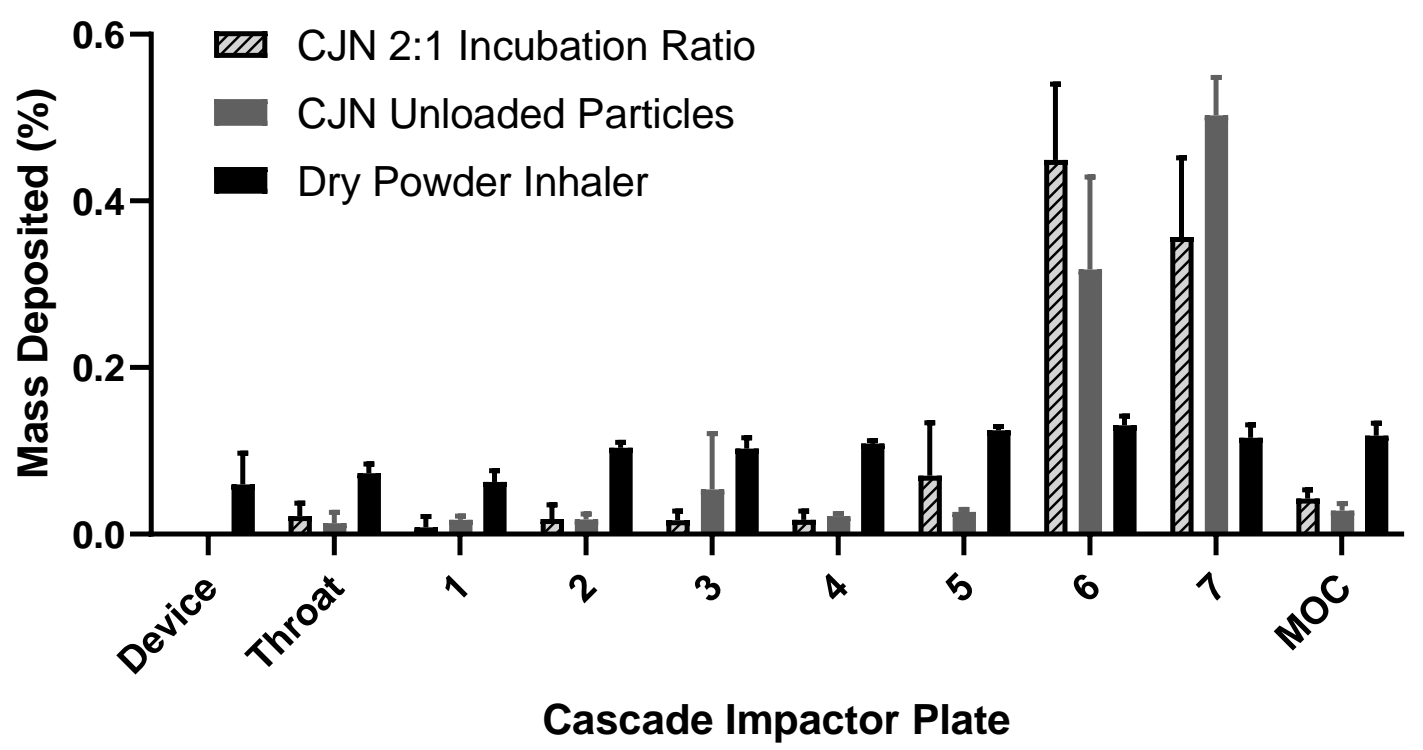

Figure S11: Mass deposition profiles for $12 \%$ defective UiO-66 using a Next Generation Impactor. The profiles are shown above for aerosolization with the CJN for loaded and unloaded NPs as well as for aerosolization using a DPI.

\section{5\% Defective Deposition Profile}

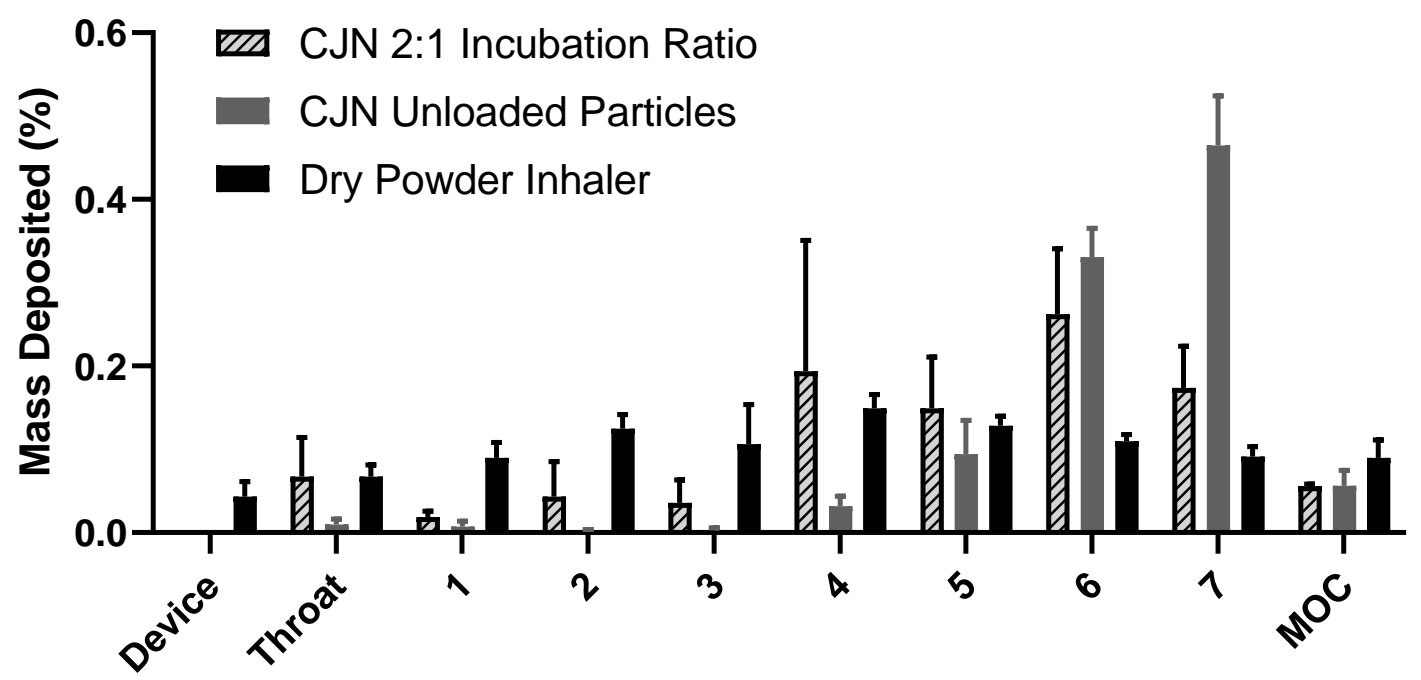

Cascade Impactor Plate

Figure S12: Mass deposition profiles for $15 \%$ defective UiO-66 using a Next Generation Impactor. The profiles are shown above for aerosolization with the CJN for loaded and unloaded NPs as well as for aerosolization using a DPI. 


\section{4-hr Cell Viability}
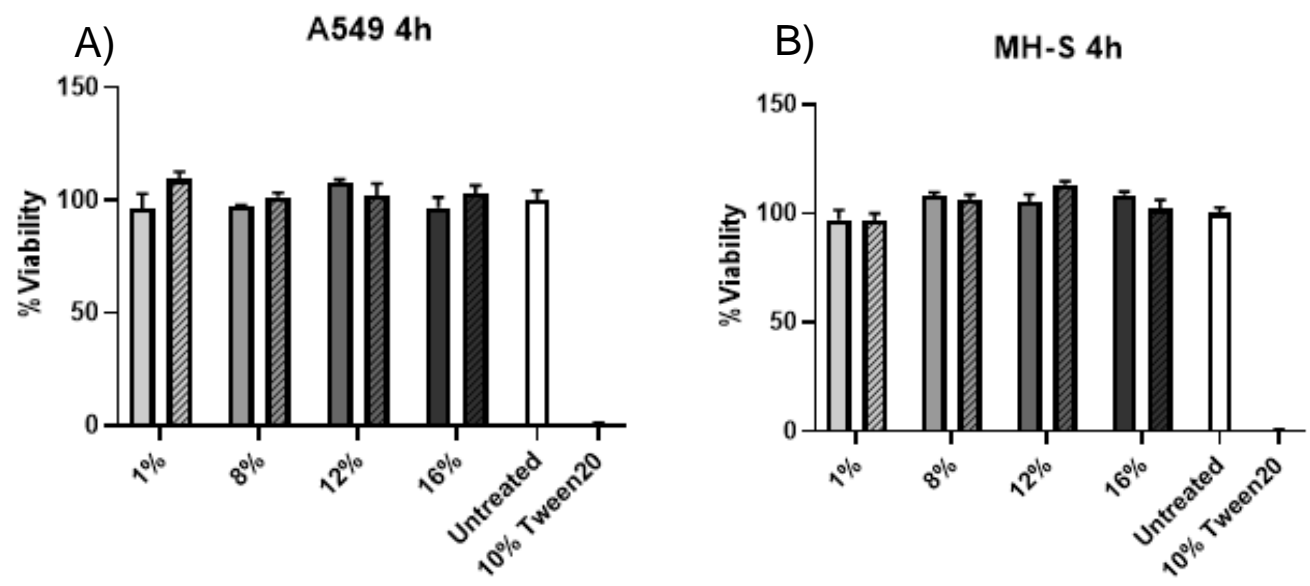

Figure S13: Cell Viability of cell lines following UiO-66 NP treatment. 4 h cell viability of A) A549 and B) $\mathrm{MH}-\mathrm{S}$ cell lines. Solid bars in the $1 \%, 8 \%, 12 \%$, and $15 \%$ conditions represent treatment with $1 \mu \mathrm{g} / \mathrm{ml}$ of UiO-66 NPs, while patterned bars represent treatment with $50 \mu \mathrm{g} / \mathrm{ml}$ of UiO-66 NPs. Bars represent the mean and error bars represent standard error $(n=3)$. All groups are not statistically significant compared to the Untreated conditions ( $\mathrm{p}>0.05$ ) as determined by Dunnett's multiple comparisons test as part of a twoway ANOVA. 


\section{Trypan Blue Quenching}

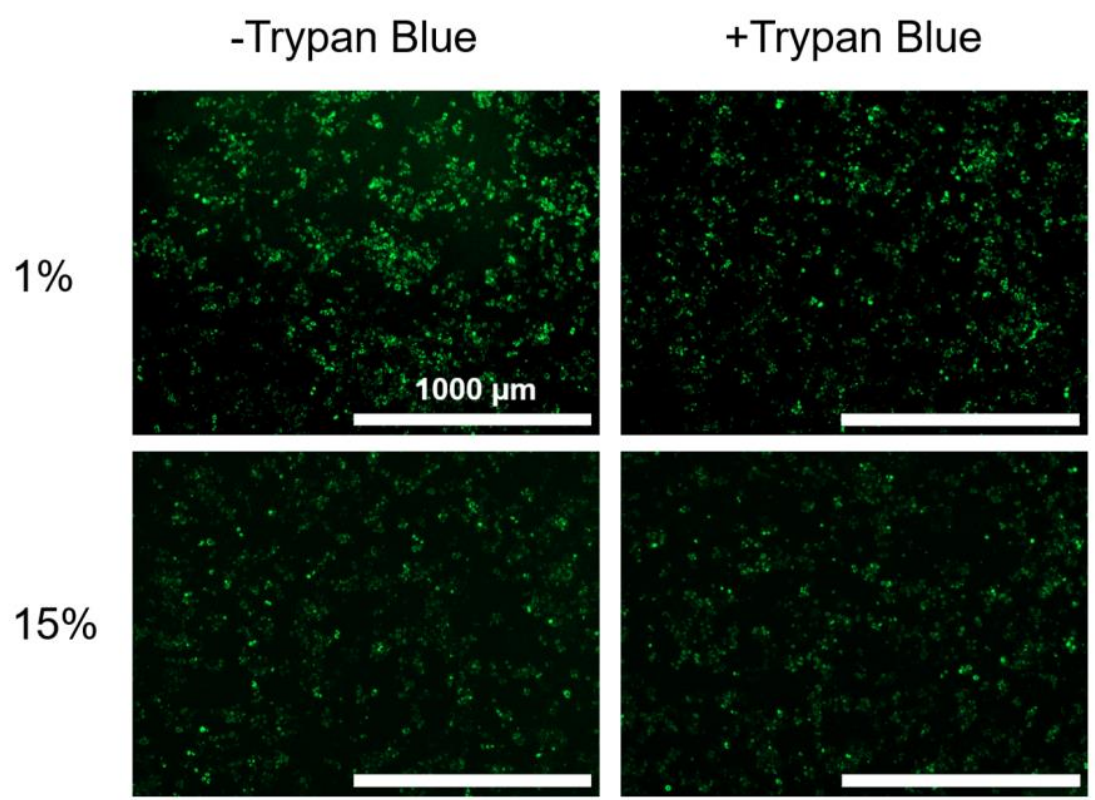

Figure S14: Trypan Blue Quenching of Free and Surface-Bound FITC-Loaded UiO-66 NPs.

Fluorescent imaging (4x) of MH-S cells treated for $24 \mathrm{~h}$ with $50 \mu \mathrm{g} / \mathrm{mL}$ FITC-loaded UiO-66 NPs of 1\% defectiveness or $15 \%$ defectiveness. Trypan Blue was used to quench FITC signal from free and cell surface-bound NPs leaving behind internalized NPs only. 


\section{4-hr Inflammatory Cytokines}

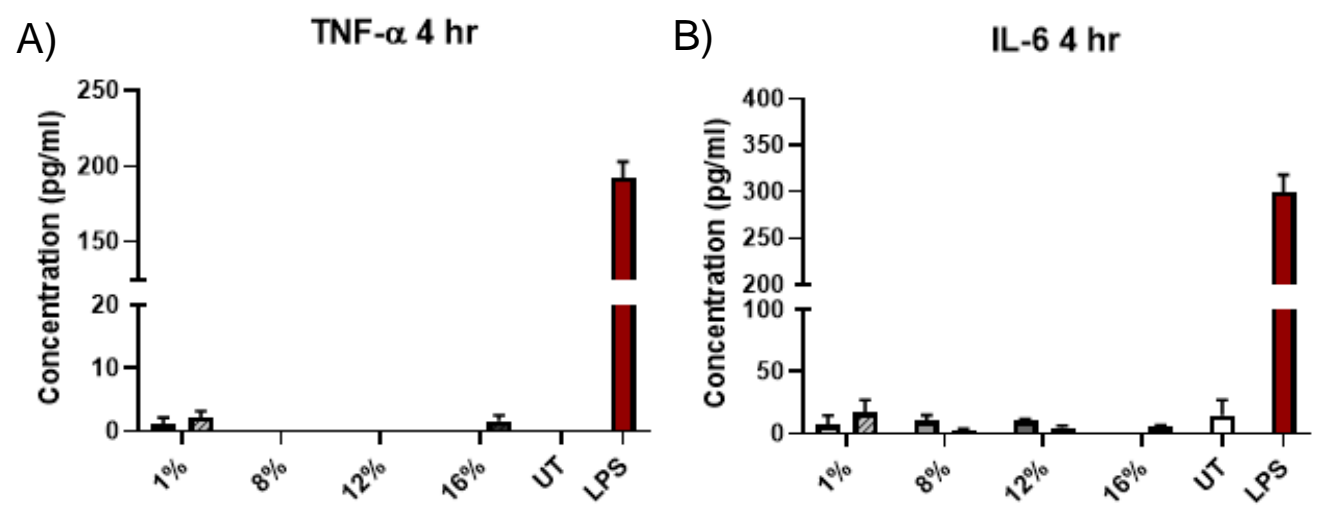

Figure S15: Inflammatory Cytokine Production in MH-S cells. $4 \mathrm{~h}$ secretion of A) TNF- $\alpha$ and B) IL-6. Solid bars in the $1 \%, 8 \%, 12 \%$, and $15 \%$ conditions represent treatment with $1 \mu \mathrm{g} / \mathrm{ml}$ of UiO-66 NPs, while patterned bars represent treatment with $50 \mu \mathrm{g} / \mathrm{ml}$ of UiO-66 NPs. Bars represent the mean and error bars represent standard error $(n=3)$. All groups are not statistically significant compared to the Untreated conditions ( $\mathrm{p}>0.05)$ as determined by Dunnett's multiple comparisons test as part of a two-way ANOVA. 


\section{BALF Flow Cytometry Gating}
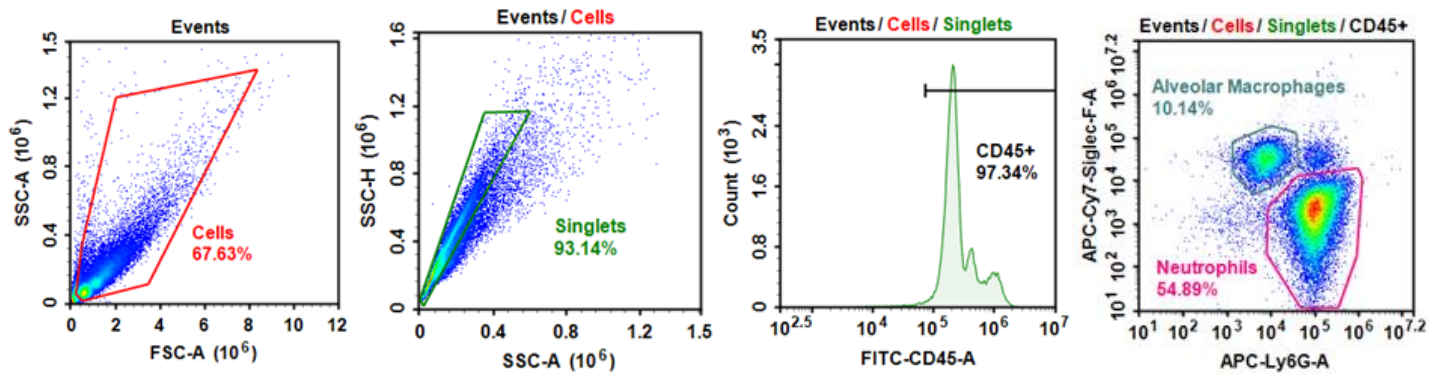

Figure S16: Representative Flow Cytometry Gating of BALF. Cells in the BALF were separated from debris and single cell populations were identified. CD45+ events were gated on a Siglec-F and a Ly6G plot; alveolar macrophages were identified as CD45+/Ly6G-/Siglec-F+ and neutrophils were identified as CD45+/Ly6G+/Siglec-F-. 


\section{SI References}

1. Pelfrêne A, Cave MR, Wragg J, Douay F. In Vitro Investigations of Human Bioaccessibility from Reference Materials Using Simulated Lung Fluids. Int J Environ Res Public Health. 2017 Jan 24;14(2):112. https://pubmed.ncbi.nlm.nih.gov/28125027 